QUELQUES MOTS SUR LES MOEURS DE L'HYLA VERSICOLOR DAUDIN

ET SUR LACCOUPLEMENT DES BATRACIENS ANOURES

\title{
par HÉRON-ROYER.
}

En 1889, un ami, amateur de Batraciens, me fit présent, à son retour d'Amérique, d'un couple de Hyla versicolor qu'il s'était procuré à New-York.

Ces deux Rainettes étaient à peu près de la taille de notre Hyla arborea. Toutes deux étaient fort tristes à leur arrivée, enfouies dans la terre trop sèche que contenait la boìte de transport; mais, avec quelques soins, je les vis revenir à la santé. Leur coloration grise devint plus franche et leur peau moins flasque, puis les taches ou dessins foncés qui agrémentent le dessus du corps et des membres prirent un ton plus vif et devinrent mieux caractérisés. En mai, lorsque l'appétit leur vint, les flancs de la femelle présentèrent une légère teinte de vert extrêmement tendre; plus tard, cette teinte chatoyante s'étendit davantage, et, suivant le beau temps et l'impression de l'animal, elle apparaissait jusque sur la face externe des cuisses.

Le mâle montrait toujours des couleurs un peu plus sombres, ne sortant pas des teintes grises, avec les dessins semblables à ceux de la femelle en teinte foncée ; l'ensemble de sa coloration s'harmonisait si bien avec les branches d'arbres qui lui servaient de perchoir, qu'il m'est arrivé souvent de le chercher longtemps avant de le découvrir.

Les deux sexes, comme cela se voit chez cette espèce, ont le dessous des cuisses et le pli de l'aîne d'un beau jaune soufre marbré ou piqueté de brun, ce qui les aide encore à se dissimuler aux regards et leur donne assez exactement l'apparence d'une des sinuosités de l'écorce où le jaune mèlé de brun simule assez bien des Champignons inférieurs. Ajoutons à cela, qu'avec leur robe bariolée de divers gris, ces Rainettes ont constamment la peau granuleuse, ce qui, probablement, leur a valu le nom de Crapaud d'arbre, sous lequel on les désigne en Amérique.

A peine étais-je en possession de ce couple d'Hyla, que je désirais les voir se reproduire. Je ne ménageai rien pour cela : cage spacieuse, eau fraîche et nourriture abondante. Mais toutes mes prodigalités furent sans succès. Néanmoins, j'ai relevé de bonnes observations, dont une surtout, l'accouplement, touche de près à la question. 
Mon attention fut attirée d'abord par l'influence pathologique du contact de ces Rainettes sur les autres Batraciens du mème genre, qui étaient réunis dans la cage où je les retenais prisonnières: Hyla arborea, H. Savignyi et $H$. barytonus formaient ensemble un contingent de quarante-quatre individus. Toutes étaient, au début de cette expérience, en parfaite santé : les premières étaient de réceute capture; les secondes avaient été élevées par moi en captivité et entraient dans leur cinquième année; quant aux troisièmes, elles n'étaient captives que depuis quinze mois environ et leur embonpoint ne laissait rien à désirer.

Ce ne fut que deux mois après avoir introduit auprès des trois autres espèces le couple d'Hyla versicolor, que je m'aperçus d'un changement fâcheux dans la santé de ces dernières. Peu à peu j'en vins à constater que presque toutes mes Rainettes étaient atteintes d'un amaigrissement général. Contrairement à ce qui avait lieu chaque année, au mois de mai, les chants furent presque nuls et lorsque les mâles émettaient leur appel, on sentait que cette expression très affaiblie n’indiquait point le réel besoin de la reproduction; les femelles étaient alanguies par la mème cause. Bientôt il y eut des morts et tous mes soins furent impuissants pour arrèter l'épidémie qui sévissait sur cette petite famille, tandis que, dans les cages voisines, tous mes pensionnaires avaient conservé leur état normal.

Enfin, en moins d’une année, j’ai compté quarante-deux victimes, il ne restait donc que les deux Américaines, ce qui m’amena à conclure que l'absorption du venin cutané de l'Hyla versicolor était la seule cause de l'empoisonnement de ses congénères européennes.

Les symptòmes de la mort ont été un amaigrissement général et une sorte de dessiccation anticipée de la peau, laquelle était alors rétrécie et collée aux muscles ; lorsque la mort survenait, l'abdomen restait ferme sans ètre gonflé d'air, et le dessous des cuisses avait une teinte sanguine, non toujours uniforme, mais souvent jaspée. Chez d'autres sujets, la teinte sanguine affectait le péritoine et tout le membre pelvien; chez d'autres, et surtout chez Hyla arborea, cette teinte sous-cutanée envahissait aussi l'abdomen, la gorge et le dessous des membres antérieurs.

Je ne m'arrètai pas à ces seules remarques et je continuai l'expérience sur quelques Grenouilles : Rana agilis et $R$. Latastei. Ces Batraciens, mis en compagnie du couple de $H$. versicolor, ne tardèrent pas à devenir souffreteux, puis, plus tard, à mourir dans des conditions presque identiques à celles relatées ci-dessus. Les 
Rana agilis vécurent plusieurs mois, tandis que les Rana Latastei périssaient en moins de vingt jours. Chez plusieurs sujets, on remarquait de petites plaies cancéreuses qui s'étaient formées durant ce court laps de temps et qui, chez un R. agilis surtout, s'étaient développées d'une façon effrayante, sans en excepter l'inflammation très vive des parties inférieures.

Les Crapauds, les Tritons et le Pleurodèle m’ont paru réfractaires au venin cutané de la Rainette américaine, car, après dix mois de cohabitation, ils en sortaient indemnes.

L'Hyla versicolor, contrairement à ce que jai observé jusqu'alors chez les autres Rainettes, aime à chasser les Hyménoptères : j'essayai de nourrir mes deux exemplaires avec les diverses Mouches qui venaient se faire prendre dans les pièges, mais je vis bientôt qu'ils néğligeaient la Musea domestica et n'étaient guère friands de Musca vomitoria. Je dus alors chercher de plus grosses proies, telles que des Papillons blancs (Pieris brassicae, P. rapae, P. napi), qui semblaient mieux leur plaire. Mais un jour, il se prit, dans les pièges à Mouches, un certain nombre de Guêpes attirées par des fruits sucrés : dès que ces Insectes furent introduits dans leur cage, mes deux Rainettes se jetèrent dessus avec un empressement que je ne leur avaıs jamais vu et les gobèrent à plaisir; dans la fougue de leur gloutonnerie, elles s'élançaient si violemment sur leur proie, qu'elles s'écorchaient le nez sur les parois de la cage. La chose se renouvelant chaque jour, la plaie s'agrandissait, sans toutefois modérer leur voracité pour ces Hyménoptères.

Un jour, en saisissant une Guêpe, le mâle fut piqué au-dessous de l'œil gauche. Il manifesta sa souffrance par des contorsions et en passant sa main sur son œil malade; mais cela ne fut l'affaire que de deux jours, après quoi il recommença à rechercher ces mèmes Insectes. L'œil se rouvrit peu à peu et il se guérit, sans qu'il s'y montrât aucune enflure. Le venin des Hyménoptères n'exercerait donc qu'une légère action sur ce Batracien, ce qui nous autorise à penser qu'à l'état sauvage l'Hyla versicolor poursuit de préférence ces Insectes.

L’année dernière, vers le mois de Juillet, le mâle essaya à plusieurs reprises d'émettre un chant ; mais son cri était si incertain que je ne trouvai pas utile d'en tenir compte. Cette année, vers la mème époque, il recommença sur un ton plus vigoureux et plus élevé. Ce chant, toutefois, est moins puissant que celui de notre Hyla arborea : il ressemble à un bèlement de Chèvre ou à un rire

$$
\mathrm{XV},-17
$$


saccadé, qu'on peut exprimer ainsi : bêe, ké, $\grave{e}-\grave{e}-\grave{e}-\grave{e}-\grave{e}-\grave{e}-\grave{e}$; la première syllabe longue, l'autre sur un ton plus élevé et plus bref, les suivantes en un rire automatique et accentué, quoique grave. Ce chant est tout différent de ceux de nos Rainettes européennes, il présente trois changements différents dans la voix : le bê, le ké et les ̀̀ ouverts; de plus, il est rendı comique par les eflorts répétés que fait le Batracien pour l'émettre. Ces efforts provoquent un éloranlement de toute la partie antérieure du corps, laquelle s'agite d'une façon automatique à chaque syllabe émise.

J'avoue que l'effet m'a surpris, tant il est curieux : à chaque reprise, je me sentais entraîné à rire, en même temps que plongé dans un étonnement que je ne pouvais réprimer. Voulant savoir si cette excitation à l'hilarité se reproduirait sur d'autres comme sur moi, j'ai fait venir deux personnes d'àge et d'impressionnabilité très différents : une dame de 58 ans et une fillette de 4 ans. Sans leur dire de quoi il s'agissait, je les fis passer au jardin et leur montrailes Rainettes à travers les vitres de la cage, les entretenant des variétés de coloration que présentent ces animaux, en attendant qu'il plaise au mâle de gonfler sa gorge. Cela ne tarda pas trop; il y eut de suite une marque d'étonnement; puis, dès que l'animal se mit à chanter, mes deux spectatrices partirent d'un éclat de rire irrésistible, que mes enfants et moi-même n’avons pu éviter de partager.

C'est bien là le chant d'amour. Après l'avoir rẻpété plusieurs fois, le mâle se dirige vers la femelle; mais celle-ci reste insensible à son appel et ne quitte pas la branche, sur laquelle elle est comme incrustée.

Le màle vient paisiblement se cramponner sur son dos : il lui pose ses mains sur les épaules; puis, comme fait le mâle chez Hyla barytonus (1), il s'y maintient solidement, en tenant, entre son pouce et son index repliés, la saillie latérale que présente l'épaule. Les deux autres doigts restent étendus et se fixent à l'aide de leurs disques d'adhérence, l'un en avant du bras sur le côté du cou, près de l'oreille, le dernier sur le haut de l'humérus.

Lorsque, pour la première fois, le 13 mai 1884, je fis connaître à la Société Zoologique de France ce nouvel accouplement chez les Anoures, je le croyais propre à la Rainette baryton, mais, trois ans après, j'eus à signaler un accouplement presque identique chez Bufo musicus (2).

(1) Bull. de la Soc. Zool. de France, IX, 1884.

(2) Notices sur les mours des Batraciens. Bull. de la Soc. d'études scientif. d'Angers, XVI, 1886. 
Il n'est pas sans intérêt, en terminant ce travail, de rappeler brièvement le mode de rapprochement sexuel chez les vingt-six espèces ou variétés d'Anoures qu'il m'a été possible d'observer depuis une douzaine d'années, afin de démontrer son étonnante diversité chez les espèces d'un mème genre. On verra dans cet exposé que l'accouplement sus-axillaire, qui fait l'objet principal de cette note, se retrouve chez une autre espèce que l'on ne soupconnait point : espèce européenne et francaise, l'Hyla Savignyi, qui se trouve abondamment en Corse et dans plusieurs autres îles de la Méditerranée et dont l'aire géographique s'étend jusqu'en Asie (1).

L'accouplement est dit pectoral, lorsque les mains du mâle s'avancent et viennent se joindre sur la poitrine de la femelle. On le rencontre chez :

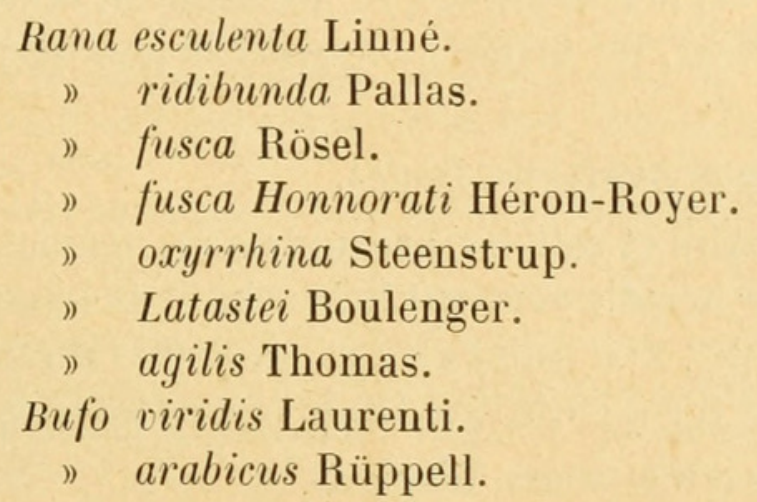

Il est axillaire, lorsque le mâle enfonce ses poings sous les aisselles de la femelle. On le rencontre chez :

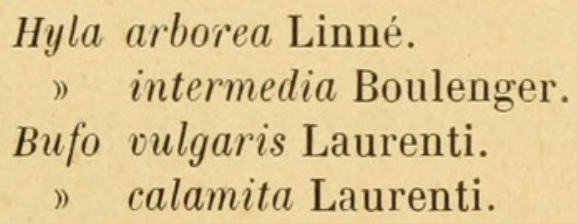

Il est sus-axillaire, lorsque le mâle tient la femelle à la saillie des épaules, comme nous l'avons expliqué longuement dans cette note. On le rencontre chez :

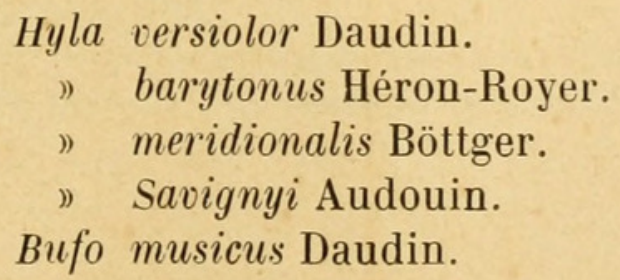

(1) Extrait d'une note inédite sur le genre Hyla, que nous devons publier prochainement. 
Il est inguinal, lorsque le mâle embrasse la femelle au bas des lombes et fixe ses mains au-dessus du pubis. On le rencontre chez :

$$
\begin{aligned}
& \text { Pelobates fuscus Laurenti. } \\
& \text { " cultripes Cuvier. } \\
& \text { Bombinator igneus Laurenti. } \\
& \text { " pachypus Fitzinger. }
\end{aligned}
$$

Il est axillo-inguinal, lorsque les mains du mâle n'ont à effectuer qu'un glissement de haut en bas sur le ventre de la femelle. On le rencontre chez :

\section{Discoglossus pictus Otth. \\ » aurilus Héron-Royer.}

Il est lombo-pubien, lorsque les coudes du mâle viennent s'appuyer sur le pubis de la femelle et que, les avant-bras étant rapprochés et dirigés en haut, les mains avoisinent le post-sternum. On le rencontre chez :

\section{Pelodytes punctatu; Dugès.}

Il est enfin lombaire et collaire, accouplement terrestre en deux temps distincts : $1^{\circ}$ le mâle soutient le bassin de la femelle, jusqu'à l'évacuation des œufs; $2^{\circ}$ il saisit celle-ci au défaut du cou pour les féconder et les fixer à ses chevilles. On le rencontre chez :

\section{Alytes obstetricans Wagler.}

Anciennement, on ne distiguait que deux sortes d'accouplement chez les Batraciens anoures, l'axillaire et le lombaire, mais, d'après les nombreuses observations que nous avons faites sur ces animaux, nous avons cru utile d'établir quelques subdivisions, qui caractérisent plus nettement le mode d'accouplement propre à chaque espèce.

A. de l'Isle appelait accouplement cervical le deuxième temps de l'accouplement de l'Alyte; nous proposons d'y substituer le nom d'accouplement collaire, qui a le mérite d'ètre plus exact (1).

(1) Pour plus amples renseignements, voir mes publications insérées dans le Bull. de la Soc. Zool. de France, tomes III, IV, VIII, IX, X, XI, XIII et XIV; Bull. de la Soc. d'études sc. d'Angers, années 1884, 1885, 1886, 1889 et 1890 ; et pour le Bufo vulgaris, Bull. de l'Acad. Roy. des sc. de Belgique, (3), X, no 11, 1885. 


\section{OUVRAges REÇUS LE 11 NOVEMBre 1890}

1. G. Cotteau, Echinides recueillis par M. Jullien sur les côtes de Guinée. Bull. de la Soc. Zool. de France, XIV, p. 340, 1889.

2. Id., Description de trois Echinides vivants recueillis par M. le $D^{\mathrm{r}} J$. Jullien sur les côtes de Guinée (Libéria). C. R. des séances du Congrès international de zoologie, p. 281, avec 3 pl., 1889.

3. Id., Congrès scientifique. Les Sciences naturelles à la réunion des délégués des Sociélés savantes. Bull. de la Soc. des sciences de l'Yonne, $4^{\mathrm{er}}$ semestre 1889.

4. Id., La géologie à l'Exposition universelle el dans les Congrès internationaux de 1889. Ibidem, $1^{\text {er }}$ semestre 1890.

5. Id., Les délégués des Soci tés savantes à la Sorbonne en 1890. Ibidem, $2^{\mathrm{e}}$ semestre 1890.

6. Id., Considérations générales sur les Echinides éocènes de la France. Association française pour l'avancement des sciences. Congrès de Paris, 1889.

7. Id., Note sur doux Echinodermes nouveaux. Bull. de la Soc. géol. de France, (3), XVII, p. 150, 1888.

8. Id., Echinides recueillis dans la province d'Aragon (Espagne), par M. Maurice Gourdon. Ibidem, (3), XVIII, p. 178, 1889.

J. Deniker, Essai d'une classification des races humaines basée uniquement sur les caractères physiques. Bull. de la Soc. d'anthropologie, 1889.

1. H. Gadeau de Kerville, La Sociélé des amis des sciences naturelles de Rouen en 1888. Compte-rendu annuel. Bull. de la Soc. des amis des sc. nat. de Rouen, $2^{\mathrm{e}}$ semestre 1888.

2. Id., Note sur la venue du Syrrhapte paradoxal en Normandie. Ibidem, avec 1 pl., A $^{\text {er }}$ semestre 1889.

3. Id., Deuxième addenda à la faune des Myriopodes de la Normandie, suivi de la description d'une variété nouvelle (var. lucida Latz.) du Glomeris marginata Villers. Ibidem.

4. Id., La Société des amis des sciences naturelles de Rouen en 1889. Compterendu annuel. Ibidem. $2^{\text {e }}$ semestre 1889.

5. Id., Sur un Levraut monstrueux du genre hétéradelphe. Le Naturaliste, 15 déc. 1889.

6. Id., Expériences tératogéniques sur différentes espèces d'Insectes. Ibidem. 1 วั mai 1890.

7. Id., Sur un cas d'amitié réciproque chez deux Oiseaux (Perruche et Sturnidé). Ibidem, $1^{\mathrm{er}}$ aoùt 1890.

8. Id., Sur l'existence du Palaemonetes varians Leach dans le déparlement de la Seine-Inférieure. Bull. de la Soc. Zool. de France, XV, p. 21, 1890. 


\section{$2 \mathrm{BHL}$ Biodiversity Heritage Library}

Herón-Royer, L-F. 1890. "Quelques mots sur les moeurs de l'Hyla versicolor Daudin et sur l'accouplement des batraciens anoures." Bulletin de la Société zoologique de France 15, 205-211. https://doi.org/10.5962/bhl.part.18724.

View This Item Online: https://www.biodiversitylibrary.org/item/23564

DOI: https://doi.org/10.5962/bhl.part.18724

Permalink: https://www.biodiversitylibrary.org/partpdf/18724

\section{Holding Institution}

MBLWHOI Library

\section{Sponsored by}

MBLWHOI Library

\section{Copyright \& Reuse}

Copyright Status: NOT_IN_COPYRIGHT

This document was created from content at the Biodiversity Heritage Library, the world's largest open access digital library for biodiversity literature and archives. Visit BHL at https://www.biodiversitylibrary.org. 